\title{
Transtorno de estresse pós-traumático como acidente de trabalho em um bancário: relato de um caso
}

\author{
Erika Bucasio* \\ Isabela Vieira* \\ William Berger** \\ Dulcéa Martins*** \\ Carmelita Souza**** \\ Deborah Maia** \\ Ivan Figueira† \\ Sílvia Jardim††
}

\section{INTRODUÇÃO}

O diagnóstico de transtorno de estresse pós-traumático (TEPT) surgiu pela primeira vez numa classificação psiquiátrica oficial em 1980", tendo sido estudado em vários países e populações ${ }^{2}$. É um transtorno prevalente, crônico, incapacitante, resistente ao tratamento e pouco diagnosticado. Está associado a alcoolismo, suicídio, insatisfação no trabalho, absenteísmo e aposentadoria precoce $e^{3-6}$.

Situações traumáticas no ambiente de

\footnotetext{
* Médica Psiquiatra. Projeto de Atenção à Saúde Mental dos Trabalhadores (PRASMET). Programa de Transtornos Mentais relacionados ao Estresse. Instituto de Psiquiatria, Universidade Federal do Rio de Janeiro, RJ.

** Médico(a) Psiquiatra. Programa de Transtornos Mentais relacionados ao Estresse. Instituto de Psiquiatria, Universidade Federal do Rio de Janeiro, RJ.

*** Assistente Social. Doutoranda da Escola de Serviço Social da Universidade Federal do Rio de Janeiro, RJ.

**** Enfermeira. Mestre em Enfermagem. Instituto de Psiquiatria, Universidade Federal do Rio de Janeiro, RJ.

† Professor Adjunto do Instituto de Psiquiatria da Universidade Federal do Rio de Janeiro, RJ.

†† Doutora em Psiquiatria. Coordenadora do Projeto de Atenção à Saúde Mental dos Trabalhadores (PRASMET). Instituto de Psiquiatria, UFRJ.
}

trabalho devido à violência urbana vêm ocorrendo com maior freqüência. Entre elas, os assaltos a agências bancárias ${ }^{7}$. Em 1986, observou-se que $37,5 \%$ das vítimas de assalto a mão armada apresentaram TEPT ${ }^{8}$. Vários estudos sobre assaltos a banco mostram como esses eventos afetam a saúde mental e o desempenho no trabalho ${ }^{9-13}$. Segundo dados da Polícia Civil, no Rio de Janeiro ocorreram cinco assaltos a banco no mês de maio de 2004 e três no mesmo mês em $2003^{14}$. Dados da Secretaria de Justiça e Segurança do Rio Grande do Sul, de junho de 2003, revelam que o número de assaltos a bancos dobrou em Porto Alegre e no interior do estado ${ }^{15}$. Um percentual significativo de funcionários de agências bancárias relata nervosismo, tensão e preocupação $(66,4 \%)$ e medo de assalto $(48,6 \%)^{16}$.

O objetivo deste artigo é exemplificar, com um caso clínico, as relações entre TEPT e eventos traumáticos ocorridos no trabalho, demonstrando que TEPT pode ser uma conseqüência do acidente de trabalho. É um tema ainda pouco estudado no Brasil ${ }^{17}$, apesar dos índices elevados de violência. 


\section{APRESENTAÇÃO DO CASO}

J., sexo masculino, branco, 44 anos, $3^{\circ}$ grau completo, gerente de banco, separado, um filho.

Há três anos, J. sofreu um assalto na agência bancária onde era gerente administrativo: quatro homens armados, um deles com uma granada, entraram no banco, ameaçaram-no e agrediram-no fisicamente. Optou por abrir o cofre que tinha mais dinheiro por medo de ser morto. Teve uma arma colocada dentro de sua boca, e ao final, foi trancado no cofre, onde ficou por cerca de uma hora. Após o assalto, sem ser socorrido, teve de ir sozinho à delegacia.

Nos dias subseqüentes, para conseguir trabalhar, passava na roleta da agência e voltava várias vezes, muito sobressaltado, tremia e sentia calafrios. Emagreceu $12 \mathrm{~kg}$ em 2 meses. Desde então, não consegue mais permanecer em locais fechados nem utilizar elevador, mesmo tendo de subir mais de dez andares. Não dirige mais. Passou a sentir-se em permanente estado de alerta, com a impressão de estar sendo seguido ou de que poderia acontecer-lhe alguma coisa a qualquer momento; sente-se ameaçado, principalmente por homens negros (um dos assaltantes era negro). O coração fica acelerado, tem dificuldade de respirar e mal-estar. Refere pesadelos freqüentes sobre o assalto e temas relacionados. Tem sensação de insegurança quando anoitece, por isso evita sair à noite. Após o assalto, ficou descuidado com a aparência, distanciou-se de amigos e familiares, não saía de casa, tinha crises de choro, dificuldade de concentração e ideação suicida.

Em junho de 2002, foi encaminhado para tratamento psiquiátrico. O psiquiatra emitiu uma Comunicação de Acidente de Trabalho (CAT), a qual não foi aceita pelo banco. Foi então encaminhado ao Núcleo de Saúde do Trabalhador da Secretaria Municipal de Saúde do Rio de Janeiro (NUSAT) e ao Sindicato dos Bancários, e a seguir, para o Projeto de Atenção à Saúde Mental dos Trabalhadores do Instituto de Psiquiatria da Universidade Federal do Rio de Janeiro (PRASMET/IPUB/UFRJ), para avaliação do nexo causal entre o quadro clínico apresentado e o trabalho.

Atualmente, apresenta melhora do humor, porém ainda tem medo de locais fechados, insegurança, principalmente quando está sozinho. Às vezes, tem a sensação de que pode estar sendo seguido. Não consegue passar perto de carros-fortes ou agências bancárias. Tem pesadelos e não consegue mais dirigir. Faz tratamento psiquiátrico e psicoterápico (terapia cognitivo-comportamental) e está em uso de cloridrato de fluoxetina $40 \mathrm{mg} / \mathrm{dia}$, mirtazapina $30 \mathrm{mg} / \mathrm{dia}$, alprazolam $3 \mathrm{mg} / \mathrm{dia}$, risperidona 2 $\mathrm{mg} / \mathrm{dia}$, flurazepam $30 \mathrm{mg} / \mathrm{dia}$ e tratamento de hipertensão arterial sistêmica e prolapso de valva mitral. Recebe auxílio-doença desde outubro de 2002.

Apresenta antecedentes familiares de hipertensão arterial sistêmica e cardiopatia.

\section{DISCUSSÃO}

Esse quadro clínico caracteriza o Transtorno de Estresse Pós-Traumático (TEPT), cuja tríade de dimensões psicopatológicas são: 1) revivescência do trauma; 2) esquiva a estímulos que relembrem o trauma e distanciamento afetivo; e 3) hiperestimulação autonômica ${ }^{18,19}$.

Esse caso de TEPT foi classificado como relacionado ao trabalho porque o trauma que 0 causou caracteriza-se como acidente de trabalho, que pode ser assim definido: "(...) é o que ocorre pelo exercício do trabalho a serviço da empresa (...), provocando lesão corporal ou perturbação funcional que cause a morte, a perda ou redução da capacidade para o trabalho, permanente ou temporária"; "(...) sofrido pelo segurado no local e no horário de trabalho, em conseqüência de ato de agressão, sabotagem ou terrorismo praticado por terceiro ou companheiro de trabalho"20.

Segundo o Manual de Manejo da Lista de Doenças Relacionadas ao Trabalho, em "trabalhadores que sofrem situações descritas na definição da doença, em circunstâncias de trabalho, o diagnóstico de TEPT, excluídas outras causas não ocupacionais, permite enquadrar esta doença no Grupo I da Classificação de Schilling, ou seja, o 'trabalho' ou a 'ocupação' desempenham o papel de 'causa necessária'. Sem eles, seria improvável que o trabalhador desenvolvesse esta doença"21,22.

Indica-se a emissão da CAT quando da ocorrência de um caso de TEPT relacionado ao trabalho, pois este instrumento aciona a vigilância em saúde pública, passo também importante para a prevenção primária. O reconhecimento do TEPT no contexto do trabalho bancário é importante para orientar serviços de saúde, administradores e profissionais de recursos humanos a promover a prevenção de TEPT com intervenções na organização do trabalho. Com isso, haverá maior proteção aos trabalhadores, treinamentos adequados, suporte psicológico e acesso aos serviços de saúde, visando à melhoria da qualidade de vida. 


\section{REFERÊNCIAS BIBLIOGRÁFICAS}

1. American Psychiatric Association. Diagnostic and statistical manual of mental disorders. 3rd ed. Washington: American Psychiatric Press; 1980.

2. Yehuda R. Post-traumatic stress disorder. N Engl J Med. 2002;346(2):108-14

3. Regehr C, Goldberg G, Glancy G, Knott T. Posttraumatic symptoms and disability in paramedics. Can J Psychiatry. 2002;47:953-8.

4. Breslau N, Davis GC, Andreski P, Peterson E. Traumatic events and posttraumatic stress disorder in an urban population of young adults. Arch Gen Psychiatry. 1991;48:216-22.

5. Davis GC, Breslau N. PTSD in victims of civilian trauma and criminal violence. Psychiatr Clin N Am. 1994;17:289 99.

6. Jardim S. Ética e saúde mental do trabalhador: a legitimidade do transtorno de estresse pós-traumático relacionado ao trabalho. In: Moraes T, org. Ética e psiquiatria forense. Rio de Janeiro: IPUB-CUCA; 2001. p. 57-84.

7. Richards DA. Traumatic stress at work: a public health model. Br J Guidance Counselling. 1994;22(1):51-64.

8. Tunnecliffe M, Green S. Trauma in the workplace: dealing with stress reactions after armed hold-ups. In: Morrison D, Hartley L, Kemp D, eds. Trends in the ergonomics of work. Proceedings of the 23rd Annual Conference of the Ergonomics Society of Australia, 1986.

9. Miller-Burke J, Attridge M, Fass P. Impact of traumatic events and organizational response. A study of bank robberies. J Occup Environ Med. 1999;41(2):73-83.

10. Kamphuis J, Emmelkamp P. Crime-related trauma: psychological distress in victims of bank robbery. J Anxiety Disord. 1998;12(3):199-208.

11. Harrison C, Kinner S. Correlates of psychological distress following armed robbery. J Trauma Stress. 1998;11(4):787-98.

12. Richards DA. Symptom severity, personal and socia variables after armed robbery. $\mathrm{Br} \mathrm{J}$ Clin Psychol. 2000;39(4):415-9.

13. MacDonald HA, Colotla V, Flamer S, Karlinsky H Posttraumatic stress disorder (PTSD) in the workplace: a descriptive study of workers experiencing PTSD resulting from work injury. J Occup Rehabil. 2003;13(2):63-77.

14. Polícia Civil do Estado do Rio de Janeiro. Sete tipos de crimes sofrem queda no mês de maio. Disponível em: http://www.policiacivil.rj.gov.br/noticia.asp?id=1291.

15. Federação dos Bancários. Bancários e banqueiros debatem segurança nesta sexta. Disponível em: http:// www. bancnet.com.br/artigo.asp?PRID=1576.

16. Silva Filho JF, et al. Estudo das relações entre a prevalência de doenças mentais e a organização do trabalho bancário no Município do Rio de Janeiro. In: Textos para discussão 7 - Fórum de Ciência e Cultura. Rio de Janeiro: UFRJ; 1993.

17. Glina D, Rocha L, Batista M, Mendonça M. Saúde menta e trabalho: uma reflexão sobre o nexo com o trabalho e o diagnóstico com base na prática. Cad Saude Publica. 2001;17(3):607-16.

18. American Psychiatric Association. Diagnostic and statistical manual of mental disorders. 4th ed. Washington: American Psychiatric Press; 1994.

19. Organização Mundial da Saúde. Classificação de transtornos mentais e de comportamento da CID-10: descrições clínicas e diretrizes diagnósticas. [Tradução: Dorgival Caetano]. Porto Alegre: Artes Médicas; 1997.

20. Ministério da Previdência e Assistência Social (MPAS). Regulamento dos benefícios da previdência social. Decreto ํ 2172 de 05/03/1997.
21. Ministério da Saúde. Lista de doenças relacionadas ao trabalho. Brasília: Ministério da Saúde; 2000.

22. Ministério da Saúde. Doenças relacionadas ao trabalho: manual de procedimentos para os serviços de saúde. Brasília: Ministério da Saúde; 2001

\section{RESUMO}

Este artigo relata o caso de um gerente de banco atendido por uma equipe multidisciplinar através de anamnese psiquiátrica e ocupacional e aplicação de entrevista psiquiátrica semi-estruturada (SCID) para transtorno de estresse pós-traumático (TEPT). O objetivo do artigo é exemplificar, com um caso clínico, as relações entre o TEPT e eventos traumáticos ocorridos no trabalho, demonstrando que o TEPT pode ser uma conseqüencia de acidente de trabalho bancário. A visão do TEPT como doença ocupacional é um conceito novo e relevante devido aos índices elevados de violência no Brasil. O reconhecimento do TEPT no contexto do trabalho bancário é importante para orientar serviços de saúde, administradores e profissionais de recursos humanos na prevenção de TEPT (treinamento adequado, suporte psicológico, emissão de Comunicação de Acidente de Trabalho, intervenções na organização do trabalho), visando à melhoria da qualidade de vida.

Descritores: Transtorno de estresse pós-traumático, acidente de trabalho, assalto a banco.

\section{ABSTRACT}

This article reports the case of a bank manager assessed by a multidisciplinary team using psychiatric and occupational history taking and a semi-structured interview (SCID) for post-traumatic stress disorder (PTSD). The aim of this article is to use a clinical case to illustrate the relationship between PTSD and traumatic events in the workplace, showing that $P T S D$ may result from an occupational injury. The notion of PTSD as an occupational disease is a new and relevant concept due to high levels of violence in Brazil. Acknowledging PTSD in the context of bank workers is important to guide health services, administrators, and human resource professionals in applying preventive measures (appropriate training, psychological support, filling out of Work Injury Reports and interventions in the organization of work) that will provide improved quality of life.

Keywords: Post-traumatic stress disorder, work injury, bank robbery.

Title: Post-traumatic stress disorder as a result of occupational injury: the case of a bank employee

\section{RESUMEN}

Este artículo relata el caso de un gerente de banco atendido por un equipo multidisciplinar a través 
de anamnesia psiquiátrica y ocupacional, y aplicación de entrevista psiquiátrica semi-estructurada (SCID) para trastorno por estrés postraumático (TEPT). El objetivo del artículo es ejemplificar, con un caso clínico, las relaciones entre TEPT y eventos traumáticos ocurridos en el trabajo, demostrando que el TEPT puede ser una consecuencia de accidente de trabajo bancario. La visión del TEPT como enfermedad ocupacional es un concepto nuevo $y$ relevante, debido a los índices elevados de violencia en Brasil. El reconocimiento del TEPT en el contexto del trabajo bancario es importante para orientar los servicios de salud, administradores y profesionales de recursos humanos en la prevención del TEPT (entrenamiento adecuado, soporte psicológico, emisión de comunicación de accidente de trabajo, intervenciones en el ordenamiento del trabajo), visando a la mejoría de la calidad de vida.

Palabras clave: Trastorno por estrés postraumático, accidente de trabajo, asalto a banco.

Título: Trastorno por estrés postraumático como accidente laboral en un bancario: relato de caso

\section{Correspondência:}

Erika de Paiva Bucasio

Rua Magalhães Couto, 34, casa 5, apto 201 - Méier CEP 20735-180 - Rio de Janeiro - RJ

E-mail: epbucasio@medscape.com

Copyright (C) Revista de Psiquiatria do Rio Grande do Sul - SPRS 